Nr. $210 / 96$

Generalized Solutions of Linear Parabolic Stochastic Partial Differential Equations

J. Potthoff, G. Våge and H. Watanabe 


\title{
Generalized Solutions of Linear Parabolic Stochastic Partial Differential Equations
}

\author{
Jürgen PotthofF ${ }^{1}$, Guermund VÅge ${ }^{2}$ and Hisao Watanabe ${ }^{3}$
}

\begin{abstract}
Existence and uniqueness theorems for parabolic stochastic partial differential equations with space-time white noise are proved. The method is a combination of the characterization theorem for Hida distributions with the Feynman-Kac and Girsanov formulae.
\end{abstract}

\section{INTRODUCTION}

The purpose of this paper is to show that certain stochastic partial differential equations (SPDE's) which are too singular to be solved in the more traditional frameworks have solutions which are generalized Brownian functionals in the sense of Hida. We are concerned with stochastic partial differential equations of the following two types:

$$
\frac{\partial}{\partial t} u(t, x)=L u(t, x)+\eta(t, x) u(t, x)
$$

and

$$
\frac{\partial}{\partial t} u(t, x)=L u(t, x)+\sum_{i=1}^{d} \xi_{i}(t, x) \frac{\partial}{\partial x_{i}} u(t, x),
$$

where $t \in \mathbb{R}_{+}, x=\left(x_{1}, \ldots, x_{d}\right) \in \mathbb{R}^{d}, \eta(t, x), \xi_{i}(t, x),(i=1,2, \ldots, d)$, are white noise random fields with parameters $(t, x) \in \mathbb{H}=\mathbb{R}_{+} \times \mathbb{R}^{d}$, and $L$ is a uniformly elliptic second order operator.

We will understand and solve these equations in the framework of white noise analysis (see, e.g., [6]). The main idea is to take the $S$-transform (see below) of the equations, to obtain the following deterministic partial differential equations respectively:

$$
\frac{\partial}{\partial t} v(t, x)=L v(t, x)+h(t, x) v(t, x)
$$

\footnotetext{
${ }^{1}$ Lehrstuhl für Mathematik V, Universität Mannheim. Partially supported by the DFG.

${ }^{2}$ Lehrstuhl für Mathematik V, Universität Mannheim. Supported by the DFG.

${ }^{3}$ Department of Applied Mathematics, Faculty of Science, Okayama University of Science.
} 
and

$$
\frac{\partial}{\partial t} v(t, x)=L v(t, x)+\sum_{i=1}^{d} h_{i}(t, x) \frac{\partial}{\partial x_{i}} v(t, x),
$$

where $h$ and $h_{i}(i=1, \ldots, d)$ are elements in $\mathcal{S}\left(\mathbb{R}^{d+1}\right)$, and $v$ denotes the $S$-transform of $u$.

By considering the diffusion process associated with the generator $L$ we obtain the usual stochastic representation formulae for the solutions of (1.3) and (1.4). These formulae together with the characterization theorem of Hida distributions (see, e.g., $[6,8]$ ), and one of its corollaries (Lemma A.3 in [11]) will be used to prove that $v(t, x)$ and its partial derivatives are the $S$-transforms of certain generalized Brownian functionals $u(t, x)$ and their corresponding weak partial derivatives in the sense of white noise calculus. In this way we obtain solutions of (1.1) and (1.2). For equation (1.2) we also show that the solution has a representation by a generalized Feynman-Kac formula.

The SPDE's (1.1) and (1.2) arise in several contexts in mathematical physics. The Burgers equation with white noise is reduced to the SPDE (1.1) by the Cole-Hopf transformation. SPDE's of the type (1.2) were proposed in [2] as a model for the turbulent transport of a substance. The SPDE (1.1) was also discussed by D. Nualart and M. Zakai [10] and by H. Holden, T. Lindstr $\varnothing \mathrm{m}$, B. Øksendal, J. Ubøe, and T.-S. Zhang [7] from different points of view. An SPDE related to (1.2) has been considered by R. Mikulvicus and B.L. Rozovskii in [9].

Acknowledgement. H. W. acknowledges gratefully the warm hospitality and support of Mannheim University. J. P. acknowledges gratefully the warm hospitality and support of Okayama University of Science. Two of us (J.P. and G.V.) wish to thank F.E. Benth and Th. Deck for fruitful discussions.

\section{Preliminaries}

Let $W$ denote the Wiener random measure on $\left(\mathbb{R}^{d+1}, \mathcal{B}\left(\mathbb{R}^{d+1}\right)\right)$ over a probability space $(\Omega, \mathcal{B}, P)$ : For every sequence $\left\{B_{i}, i=1, \ldots, n\right\}$ of Borel subsets of $\mathbb{R}^{d+1}, W\left(B_{1}\right), \ldots, W\left(B_{n}\right)$ is a Gaussian family of centered random variables with covariance matrix $\left(\left|B_{i} \cap B_{j}\right|, i, j=\right.$ $1, \ldots, n)$, where $|\cdot|$ denotes the Lebesgue measure. The canonical realization of $W$ is given by the white noise probability space $\left(\mathcal{S}^{\prime}\left(\mathbb{R}^{d+1}\right), \mathcal{B}, \mu\right)$, where $\mathcal{B}$ is the weak Borel $\sigma$-algebra of $\mathcal{S}^{\prime}\left(\mathbb{R}^{d+1}\right)$, and $\mu$ the centered Gaussian measure whose covariance is given by the inner product of $L^{2}\left(\mathbb{R}^{d+1}\right)$. Let $X$. denote the canonical coordinate process on $\mathcal{S}\left(\mathbb{R}^{d+1}\right), X_{h}(\omega)=$ $\langle\omega, h\rangle, h \in \mathcal{S}\left(\mathbb{R}^{d+1}\right), \omega \in \mathcal{S}^{\prime}\left(\mathbb{R}^{d+1}\right)$. We can extend $X$. continuously in $L^{2}(\mu)$ to $L^{2}\left(\mathbb{R}^{d+1}\right)$, and then realize $W(B), B \in \mathcal{B}\left(\mathbb{R}^{d+1}\right)$ by $X_{1_{B}}$ on $\left(\mathcal{S}^{\prime}\left(\mathbb{R}^{d+1}\right), \mathcal{B}, \mu\right)$. From now on we shall work with this representation for $W$.

Every random variable $F \in L^{2}(\mu)$ is represented as

$$
F=\sum_{n=0}^{\infty} F_{n}
$$


(convergence in $L^{2}(\mu)$-sense) where the $F_{n}$ are orthogonal random variables of the form

$$
F_{n}=\int_{\left(\mathbb{R}^{d+1}\right)^{n}} \cdots \int_{n} f_{n}\left(a_{1}, \ldots, a_{n}\right) W\left(d a_{1}\right) \cdots W\left(d a_{n}\right),
$$

and the deterministic functions $f_{n}$ are symmetric $L^{2}\left(\left(\mathbb{R}^{d+1}\right)^{n}\right)$ kernels. Therefore we have the isomorphism

$$
L^{2}(\mu) \cong \bigoplus_{n=0}^{\infty} L_{\mathrm{sym}}^{2}\left(\left(\mathbb{R}^{d+1}\right)^{n}\right)
$$

i.e.,

$$
F \leftrightarrow\left(f_{0}, f_{1}, \ldots, f_{n}, \ldots\right)
$$

which is called the Wiener chaos expansion. It turns out that

$$
\|F\|_{2}^{2}=\sum_{n=0}^{\infty} n !\left|f^{(n)}\right|_{2}^{2}
$$

where $|\cdot|_{2}$ denotes the norm of $L^{2}\left(\left(\mathbb{R}^{d+1}\right)^{n}\right), n \in \mathbb{N}$.

The $S$-transform of $F$ is defined as follows: for $h \in \mathcal{S}\left(\mathbb{R}^{d+1}\right)$, we set

$$
S F(h):=\mathbb{E}\left(F: e^{X_{h}}:\right),
$$

with the abbreviation

$$
\begin{aligned}
: e^{X_{h}}: & =\left(\mathbb{E} e^{X_{h}}\right)^{-1} e^{X_{h}} \\
& =e^{X_{h}-\frac{1}{2}|h|_{2}^{2}}
\end{aligned}
$$

Then we obtain

$$
S F(h)=\sum_{n=0}^{\infty} \int_{\left(\mathbb{R}^{d+1}\right)^{n}} \ldots \int_{n} f_{n}\left(a_{1}, \ldots, a_{n}\right) h\left(a_{1}\right) \cdots h\left(a_{n}\right) d a_{1} \cdots d a_{n} .
$$

If we consider the last expression as a function of $\left(f_{0}, f_{1}, \ldots, f_{n}, \ldots\right)$, we observe that it extends to larger spaces than $\bigoplus_{n=0}^{\infty} L_{\text {sym }}^{2}\left(\left(\mathbb{R}^{d+1}\right)^{n}\right)$. This observation leads to spaces of generalized random variables. Here we shall only need the space of Hida distributions $(\mathcal{S})^{*}$, which we describe next.

Let $A$ be the self-adjoint extension of the differential operator

$$
\Delta h(a)+|a|^{2} h(a), \quad h \in \mathcal{S}\left(\mathbb{R}^{d+1}\right), a \in \mathbb{R}^{d+1},
$$

to $L^{2}\left(\mathbb{R}^{d+1}\right)$. Let $(\mathcal{S})$ denote the subspace of $L^{2}(\mu)$ consisting of $\varphi$ corresponding to $\left(f_{n}, n \in \mathbb{N}_{0}\right)$ so that

$$
\sum_{n=0}^{\infty} n !\left|\left(A^{\otimes n}\right)^{p} f_{n}\right|_{2}^{2}<+\infty
$$


for all $p \in \mathbb{N}$. $(\mathcal{S})$ carries a natural Fréchet topology, and $(\mathcal{S})^{*}$ is the corresponding dual. Thus we get the triple

$$
(\mathcal{S}) \subset L^{2}(\mu) \subset(\mathcal{S})^{*}
$$

(where we have identified $L^{2}(\mu)$ with its dual). $\Phi \in(\mathcal{S})^{*}$ is in one-to-one correspondence with a sequence $\left(T_{0}, T_{1}, \ldots, T_{n}, \ldots\right)$ of symmetric elements $T_{n}$ in $\mathcal{S}^{\prime}\left(\left(\mathbb{R}^{d+1}\right)^{n}\right)$, and there exists $p \in \mathbb{N}$ so that

$$
\sum_{n=0}^{\infty} n !\left|\left(A^{\otimes n}\right)^{-p} T_{n}\right|_{2}^{2}<+\infty
$$

For such a $\Phi$, we get the following generalization of $(2.3)$ :

$$
S \Phi(h)=\sum_{n=0}^{\infty}\left\langle T_{n}, h^{\otimes n}\right\rangle, \quad h \in \mathcal{S}\left(\mathbb{R}^{d+1}\right)
$$

where $\langle\cdot, \cdot\rangle$ denotes the dual pairing between $\mathcal{S}^{\prime}\left(\left(\mathbb{R}^{d+1}\right)^{n}\right)$ and $\mathcal{S}\left(\left(\mathbb{R}^{d+1}\right)^{n}\right), n \in \mathbb{N}$.

It is easy to see that the canonical coordinate process $X_{h}=\langle\cdot, h\rangle$ extends - as an element in $(\mathcal{S})^{*}$ - to $h \in \mathcal{S}^{\prime}\left(\mathbb{R}^{d+1}\right)$. The generalized random field $a \mapsto\left\langle\cdot, \delta_{a}\right\rangle \in(\mathcal{S})^{*}, a \in$ $\mathbb{R}^{d+1}$, is a white noise on $\mathbb{R}^{d+1}$, and we shall denote this field by $\eta$. Its $S$-transform is given by $S(\eta(a))(h)=h(a)$. Informally, $\eta$ can be thought of as the Lebesgue density of $W$. Similarly, the normalized exponential : $e^{\eta(a)}:$ belongs to $(\mathcal{S})^{*}$ for every $a \in \mathbb{R}^{d+1}$, and

$$
S\left(: e^{\eta(a)}:\right)(h)=e^{h(a)}, \quad h \in \mathcal{S}\left(\mathbb{R}^{d+1}\right)
$$

We usually write $a \in \mathbb{R}^{d+1}$ as $a=(t, x), t \in \mathbb{R}, x \in \mathbb{R}^{d}$, where $t$ represents time and $x$ is a space variable. Consider a mapping $u$ from $\mathbb{R}^{d+1}$ into $(\mathcal{S})^{*}:(t, x) \mapsto u(t, x)$. Under very mild conditions on the mapping (e.g., as in Chapter 8 of [6]), the expression

$$
\int_{\mathbb{R}} S u(t, x)(h) h(t, x) d t, \quad h \in \mathcal{S}\left(\mathbb{R}^{d+1}\right)
$$

is the $S$-transform of an element in $(\mathcal{S})^{*}$, which we denote by

$$
\int_{\mathbb{R}} u(t, x) \eta(t, x) d t
$$

(2.4) is called the Hitsuda-Skorokhod integral of $u$. (Actually, the "product" of $u$ and $\eta$ under the integral sign is the so-called Wick product.) It is known that when $d=0$ the Hitsuda-Skorokhod integral is a generalization of the Itô integral.

\section{Formulation of the Cauchy Problems}

We denote $\mathbb{H}=\mathbb{R}_{+} \times \mathbb{R}^{d}$ and $\mathbb{H}_{T}=(0, T) \times \mathbb{R}^{d}, T>0$, with typical elements $(t, x),(s, y)$ etc. Partial derivatives with respect to the space variable in $\mathbb{R}^{d}$ are denoted by $D_{i}, i=$ 
$1, \ldots, d$, the time derivative by $D_{t}$, and - if convenient - also by $D_{0}$. Throughout the paper, we consider a partial differential operator $L$ in $\mathbb{R}^{d}$ of the form:

$$
(L f)(x)=\frac{1}{2} \sum_{i, j=1}^{d} a_{i j}(x)\left(D_{i} D_{j} f\right)(x)+\sum_{i=1}^{d} b_{i}(x)\left(D_{i} f\right)(x), \quad f \in C^{2}\left(\mathbb{R}^{d}\right), x \in \mathbb{R}^{d}
$$

satisfying the following hypothesis:

(H1) $L$ is uniformly elliptic, i.e., there exists $\varepsilon>0$ so that for all $x, y \in \mathbb{R}^{d}$,

$$
\sum_{i, j=1}^{d} a_{i j}(x) y_{i} y_{j} \geq \varepsilon|y|^{2}
$$

(H2) For all $i, j=1, \ldots, d, a_{i j}$ and $b_{i}$ belong to $C_{b}^{2}\left(\mathbb{R}^{d}\right)$ (i.e., are bounded and have bounded, continuous derivatives up to second order).

We remark that the method of the paper applies also to problems where $L$ has time dependent coefficients. For simplicity of the presentation we refrain from considering this more general situation.

Let $u$ be a mapping from $\mathbb{H}\left(\right.$ or $\left.\mathbb{H}_{T}\right)$ into $(\mathcal{S})^{*}$. The weak derivative $D_{i} u, i=0,1, \ldots, d$, of $u$ is defined as follows. For $\varphi \in(\mathcal{S})$ consider the function $F_{\varphi}(t, x)=\langle u(t, x), \varphi\rangle$. If for $(t, x) \in \mathbb{H}$ and all $\varphi,\left(D_{i} F_{\varphi}\right)(t, x)$ exists, and $\varphi \mapsto\left(D_{i} F_{\varphi}\right)(t, x)$ defines a linear, continuous mapping from $(\mathcal{S})$ into $\mathbb{R}$, then we say that $u$ is weakly (partially) differentiable with respect to $D_{i}$ at $(t, x)$, and denote $D_{i} F_{\varphi}(t, x)$ by $\left\langle\left(D_{i} u\right)(t, x), \varphi\right\rangle$, with $\left(D_{i} u\right)(t, x) \in(\mathcal{S})^{*}$. Weak derivatives of higher order are defined in the obvious way.

In Sections 4 and 5 we solve the following Cauchy problems in $(\mathcal{S})^{*}$ :

$$
\begin{aligned}
D_{t} u(t, x) & =L u(t, x)+\eta(t, x) u(t, x), \\
D_{t} u(t, x) & =L u(t, x)+\xi(t, x) \cdot \nabla u(t, x), \\
u(0, x) & =f(x),
\end{aligned}
$$

respectively, where $f$ belongs to $C_{b}^{2}\left(\mathbb{R}^{d}\right)$. In (3.1) $\eta$ is space-time white noise, and in (3.2) $\xi$ is a $d$-vector of independent space-time white noise (generalized) random fields. In both equations multiplication by white noise is understood in the sense of Hitsuda-Skorokhod or - equivalently - Wick (the natural generalization of Itô's convention to generalized random processes and fields).

By a solution of the Cauchy problems $(3.1,3),(3.2,3)$ respectively, we mean a mapping $u$ from $\mathbb{H}$ or $\mathbb{H}_{T}$ into $(\mathcal{S})^{*}$, so that the weak derivatives $D_{t} u(t, x), D_{i} u(t, x), D_{i} D_{j} u(t, x)$ exist for all $i, j=1, \ldots, d$ and all $(t, x) \in \mathbb{H},\left(\mathbb{H}_{T}\right.$, resp.), and such that equations (3.1), (3.2) respectively hold. Furthermore, we require that $\lim _{t \downarrow 0} u(t, x)=f(x)$ for all $x \in \mathbb{R}^{d}$, where the limit is taken in the weak topology of $(\mathcal{S})^{*}$.

We remark that a solution of $(3.1,3)$ in the sense described above is also a solution in the weak sense which has been used in [10]. Hence our concept of solutions is stronger. 
Finally, in order to discuss uniqueness of solutions to the Cauchy problems we introduce the following additional assumption on the coefficients of $L$ :

(H3) For all $i, j=1, \ldots, d, a_{i j}$ has uniformly Hölder continuous derivatives up to second order.

For $T>0$, let $\mathcal{W}_{T}$ be the space of weakly measurable mappings $u: \mathbb{H}_{T} \rightarrow(\mathcal{S})^{*}$, for which there exist $p \in \mathbb{N}_{0}$ and $k>0$ so that

$$
\int_{0}^{T} \int_{\mathbb{R}^{d}}\|u(t, x)\|_{2,-p} e^{-k x^{2}} d x d t<+\infty .
$$

(Note that this entails Bochner-integrability of $u$ with respect to the measure $e^{-k x^{2}} d x d t$ over $\mathbb{H}_{T}$.)

\section{The Solution of $D_{t} u=L u+\eta u$.}

In this section we consider the Cauchy problem (3.1), (3.3). The main idea is as follows. By taking the $S$-transform of (3.1) we obtain a classical parabolic PDE, whose initial value problem (3.3) can be solved via the Feynman-Kac formula. Using this representation for the solution, we can prove regularity estimates which allow to invert the $S$-transform, and to show that we end up with a solution of (3.1), (3.3).

Taking (informally) the $S$-transform of equation (3.1) evaluated at $h \in \mathcal{S}\left(\mathbb{R}^{d+1}\right)$, we obtain

$$
D_{t} v(t, x ; h)=L v(t, x ; h)+h(t, x) v(t, x ; h), \quad(t, x) \in \mathbb{H}
$$

Fix $h$ for the moment. It is known (e.g., [5]) that under the conditions (H1), (H2) the initial value problem $(4.1)$ with $v(0, x ; h)=f(x), f \in C_{b}^{2}\left(\mathbb{R}^{d}\right)$, has a solution $v$ which is bounded, continuous on $[0, T] \times \mathbb{R}^{d}$ for every $T>0$, and which is continuously differentiable with respect to time and twice continuously differentiable with respect to the space variables on $(0, T) \times \mathbb{R}^{d}$. From now on we consider only this solution $v(t, x ; h)$.

Lemma 4.1. There exists a mapping $u: \mathbb{H} \rightarrow(\mathcal{S})^{*},(t, x) \mapsto u(t, x)$, which is for all $t>0$ weakly continuously differentiable in $t$ and twice weakly continuously partially differentiable in $x$. Moreover, the relations $S u(t, x)(h)=v(t, x ; h), S\left(D_{t} u(t, x)\right)(h)=$ $D_{t} v(t, x ; h), S\left(D_{i} u(t, x)\right)(h)=D_{i} v(t, x ; h), S\left(D_{i} D_{j} u(t, x)\right)(h)=D_{i} D_{j} v(t, x ; h)$ hold for all $i, j=1, \ldots, d, t>0, x \in \mathbb{R}^{d}$, and $h \in \mathcal{S}\left(\mathbb{R}^{d+1}\right)$.

Proof. It is well-known that the solution of $(4.1)$ with $v(0, x ; h)=f(x)$ can be represented by the Feynman-Kac formula, cf. e.g. [4, Theorem II.2.2]: Let $\widetilde{B}(t), t \geq 0$, be a $d$-dimensional Brownian motion on an auxiliary probability space $(\widetilde{\Omega}, \widetilde{\mathcal{B}}, \widetilde{P})$, and consider the stochastic differential equation associated with $L$,

$$
d X_{i}(t)=\sum_{j=1}^{d} \sigma_{i j}(X(t)) d \widetilde{B}_{j}(t)+b_{j}(X(t)) d t
$$


Here $\sigma_{i j}$ is a smooth function on $\mathbb{R}^{d}$ with $\sum_{k=1}^{d} \sigma_{i k}(x) \sigma_{j k}(x)=a_{i j}(x), x \in \mathbb{R}^{d}$. The solution of (4.2) with $X(0)=x \in \mathbb{R}^{d}$ is denoted by $(X(t, x), t \geq 0)$.

Now the Feynman-Kac formula for $v(t, x ; h)$ reads as follows (loc. cit.):

$$
v(t, x ; h)=\widetilde{\mathbb{E}}(f(X(t, x)) Z(t, x ; h))
$$

where

$$
Z(t, x ; h)=\exp \left(\int_{0}^{t} h(t-s, X(s, x)) d s\right)
$$

and $\widetilde{\mathbb{E}}$ denotes expectation with respect to $\widetilde{P}$.

It is obvious that $Z(t, x ; \cdot)$ and $v(t, x ; \cdot)$ have ray-entire extentions, i.e., for $h, g \in$ $\mathcal{S}\left(\mathbb{R}^{d+1}\right), z \in \mathbb{C}, Z(t, x ; z h+g)$ and $v(t, x ; z h+g)$ are well-defined, and entire in $z \in \mathbb{C}$. Moreover, we trivially get the estimate

$$
|v(t, x ; z h)| \leq|f|_{\infty} e^{t|z||h|_{\infty}} .
$$

Since $|\cdot|_{\infty}$ is a continuous norm on $\mathcal{S}\left(\mathbb{R}^{d+1}\right)$, it follows from the characterization theorem (e.g. $[6,8])$ that there exists $u(t, x) \in(\mathcal{S})^{*}$ with $S u(t, x)(h)=v(t, x ; h)$. This defines the mapping $u: \mathbb{H} \rightarrow(\mathcal{S})^{*}$ in the statement of the lemma.

In order to show that $u$ has the claimed differentiability properties, and that the partial derivatives commute with $S$ (to give $S D_{i} u(t, x)(h)=D_{i} v(t, x ; h)$ etc.) we apply Lemma A.1.3 in [11]. To this end we first have to establish that for all $f, g \in \mathcal{S}\left(\mathbb{R}^{d+1}\right), z \in$ $\mathbb{C}, v(t, x ; z h+g)$ is continuously differentiable in $t$ and twice continuously differentiable in $x$. This, however, is quite obvious from the representation (4.3) and left to the interested reader. Furthermore, we have to prove that the relevant partial derivatives of $v(t, x ; z h)$ admit a bound of the type (4.5) which is locally uniform in $(t, x)$.

We begin with $D_{t} v(t, x ; z h)$. An application of Itô's formula gives

$$
\begin{aligned}
D_{t} v(t, x ; z h)=\widetilde{\mathbb{E}}([z f(X(t, x))(h(0, X(t, x)) & \left.+\int_{0}^{t} D_{t} h(t-s, X(s, x)) d s\right)+ \\
& +L f(X(t, x))] Z(t, x ; z h)) .
\end{aligned}
$$

This equation yields immediately the following estimate

$$
\left|D_{t} v(t, x ; z h)\right| \leq\left(|z|\left(|h|_{\infty}+t\left|D_{0} h\right|_{\infty}\right)|f|_{\infty}+|L f|_{\infty}\right) e^{t|z||h|_{\infty}}
$$

which suffices to conclude that $u(t, x)$ is weakly continuously differentiable in $t$ for $t>$ $0, x \in \mathbb{R}^{d}$, and that $S D_{t} u(t, x)(h)=D_{t} v(t, x ; h)$.

Next consider $D_{i} v(t, x ; z h), i=1, \ldots, d$. From (4.3) we get the formula

$$
\begin{aligned}
D_{i} v(t, x ; z h)= & \widetilde{\mathbb{E}}\left(\left(\sum_{j=1}^{d}\left(D_{j} f\right)(X(t, x)) D_{i} X_{j}(t, x)\right.\right. \\
& \left.\left.+z f(X(t, x)) \sum_{j=1}^{d}\left(\int_{0}^{t}\left(D_{j} h\right)(t-s, X(s, x)) D_{i} X_{j}(s, x) d s\right)\right) Z(t, x ; z h)\right),
\end{aligned}
$$


and $D_{i} X_{j}, j=1, \ldots, d$, satisfies the following (linear) system of stochastic integral equations

$$
\begin{aligned}
D_{i} X_{j}(t, x)=\delta_{i j} & +\int_{0}^{t} \sum_{k, l=1}^{d}\left(D_{k} \sigma_{j l}\right)(X(s, x)) D_{i} X_{k}(s, x) d \widetilde{B}_{l}(s) \\
& +\int_{0}^{t} \sum_{k=1}^{d}\left(D_{k} b_{j}\right)(X(s, x)) D_{i} X_{k}(s, x) d s
\end{aligned}
$$

Equation (4.6) yields the following bound

$$
\begin{aligned}
\left|D_{i} v(t, x ; z h)\right| \leq( & \sum_{j=1}^{d}\left|D_{j} f\right|_{\infty} \widetilde{\mathbb{E}}\left(\left|D_{i} X_{j}(t, x)\right|\right) \\
& \left.+|z||f|_{\infty} \sum_{j=1}^{d}\left|D_{j} h\right|_{\infty} \widetilde{\mathbb{E}}\left(\int_{0}^{t}\left|D_{i} X_{j}(s, x)\right| d s\right)\right) e^{t|z||h|_{\infty}}
\end{aligned}
$$

Therefore we only have to show that the last two expectations are locally uniformly bounded in $t>0$ and $x \in \mathbb{R}^{d}$. But this follows from a standard estimation. (For example, one can consider first $\widetilde{\mathbb{E}}\left(\sum_{j=1}^{d}\left(D_{i} X_{j}(t, x)\right)^{2}\right)$, use (4.7) and apply Gronwall's lemma. The bound one obtains this way can be used to estimate the expectations in question.)

Finally, the estimation of $D_{i} D_{j} v(t, x ; z h)$ is done similarly and does not present any new difficulties. We therefore leave the details to the interested reader.

Now we are ready to prove our first main result:

Theorem 4.2. Under conditions $(\mathrm{H} 1)$, (H2) the initial value problem $(3.1,3)$ has a solution $u(t, x)$, which for every $T>0$ is a bounded, weakly continuous mapping from $[0, T] \times \mathbb{R}^{d}$ into $(\mathcal{S})^{*}$. Moreover, this solution is the inverse $S$-transform of (4.3).

Proof. Let $u(t, x)$ be defined as in Lemma 4.1, i.e., as the pre-image of the $v(t, x)$ in (4.3) under $S$.

First we show that $u:(t, x) \mapsto u(t, x) \in(\mathcal{S})^{*}$ is bounded on $[0, T] \times \mathbb{R}^{d}$ for every $T>0$ : this follows directly from inequality (4.5) and Lemma A.1.1 in [11]. Since for every $h \in \mathcal{S}\left(\mathbb{R}^{d+1}\right), v(\cdot, \cdot ; h)$ is continuous on $[0, T] \times \mathbb{R}^{d}$, it follows from Lemma A.1.2 in: [11] that $u$ is weakly continuous from $[0, T] \times \mathbb{R}^{d}$ into $(\mathcal{S})^{*}$.

Next we show that $u(t, x) \rightarrow f(x), x \in \mathbb{R}^{d}$, as $t \downarrow 0$, weakly in $(\mathcal{S})^{*}$. Since we have from (4.3) that $v(t ; x ; h) \rightarrow f(x)$ as $t \downarrow 0$ for all $h \in \mathcal{S}\left(\mathbb{R}^{d+1}\right)$, it follows that $u(t, x) \rightarrow f(x)$ on the linear span of the exponential vectors : $e^{\langle\cdot, h\rangle}$ : in $(\mathcal{S})$. Since $u(t, x)-f(x)$ is bounded in $(\mathcal{S})^{*}$ in $t \in[0, T], T>0$, the last stated convergence extends to all of $(\mathcal{S})$.

It remains to prove that (3.1) holds. The fact the $v(t, x)$ solves (4.1) means that $u(t, x)$ solves $(3.1)$ when paired with elements from the linear span of the exponential vectors. Since this span is dense in $(\mathcal{S}) ; u(t, x)$ solves $(3.1)$. 
In order to obtain a stochastic representation formula directly for $u$, we define for $(t, x) \in \mathbb{H}$ and $\widetilde{P}$-a.e. $\widetilde{\omega} \in \widetilde{\Omega}$, the mapping $T_{t, x}(\widetilde{\omega})$ from $\mathcal{S}\left(\mathbb{R}^{d+1}\right)$ into $\mathbb{R}$ by

$$
h \longmapsto\left\langle T_{t, x}(\widetilde{\omega}), h\right\rangle:=\int_{0}^{t} h(t-s, X(s, x)(\widetilde{\omega})) d s .
$$

It is obvious that $T_{t, x}$ belongs $\widetilde{P}$-a.s. to $\mathcal{S}^{\prime}\left(\mathbb{R}^{d+1}\right)$. Therefore, $: \exp \left(\left\langle\cdot, T_{t, x}\right\rangle\right):$ is $\widetilde{P}$ almost surely in $(\mathcal{S})^{*}$.

Theorem 4.3. The solution $u(t, x)$ in Theorem 4.2 has a representation given by the following generalized Feynman-Kac formula:

$$
u(t, x)=\widetilde{\mathbb{E}}\left(f(X(t, x)): e^{\left\langle\cdot, T_{t, x}\right\rangle}:\right)
$$

where the expectation in $(4.9)$ is a Bochner integral in $(\mathcal{S})^{*}$.

Proof. Let $h \in \mathcal{S}\left(\mathbb{R}^{d+1}\right)$ and consider $Z(t, x ; h)$ given in (4.4). It can be written as

$$
Z(t, x ; h)=\exp \left(\left\langle T_{t, x}, h\right\rangle\right)
$$

with $T_{t, x}$ defined in (4.8). Moreover $Z(t, x ; h)$ is (a.s.) the $S$-transform of the Hida distribution : $e^{\left\langle\cdot, T_{t, x}\right\rangle}:$. The bound $|Z(t, x ; z h)| \leq \exp \left(|t||z||h|_{\infty}\right)$ shows that we can apply Theorem 4.51 in [6] with the result that $S^{-1} f(X(t, x)) Z(t, x ; \cdot)=f(X(t, x)): e^{\left\langle\cdot, T_{t, x}\right\rangle}$ : is Bochner integrable in $(\mathcal{S})^{*}$. Furthermore, the expectation with respect to $\widetilde{P}$ and the $S$-transform can be interchanged, which proves $(4.9)$.

Note that for all $T \in \mathcal{S}^{\prime}\left(\mathbb{R}^{d+1}\right)$, the $(\mathcal{S})^{*}$-element : $\exp (\langle T, \cdot\rangle)$ : is positive (in the sense of Hida distributions, e.g., [6]). It is then obvious from formula (4.9) that $u(t, x)$ is positive if the initial condition $f$ is. For general $f \in C_{b}\left(\mathbb{R}^{d+1}\right)$, we can decompose $f$ into its positive and negative parts, and it follows from (4.9) that $u(t, x)$ is the difference of two positive Hida distributions. Applying Yokoi's theorem (e.g., Theorem 4.26 in [6]) we obtain the following result.

Corollary 4.4. For all initial conditions $f \in C_{b}\left(\mathbb{R}^{d+1}\right)$, and all $(t, x) \in \mathbb{H}$, the solution $u(t, x)$ in Theorem 4.2 is a signed measure on $\mathcal{B}$. In particular, if $f$ is positive, then $u(t, x)$ is a measure on $\mathcal{B}$.

We end this section by discussing the uniqueness of the problem $(3.1,3)$. Let $T>0$. It is well-known that under conditions $(\mathrm{H} 1),(\mathrm{H} 2)$, and $(\mathrm{H} 3)$ the initial value problem for (4.1), $v(0, \cdot)=f \in C_{b}\left(\mathbb{R}^{d}\right)$, has a unique solution in the class of functions $w$ on $[0, T] \times \mathbb{R}^{d}$ so that for some $k>0$,

$$
\int_{0}^{T} \int_{\mathbb{R}^{d}}|w(t, x)| e^{-k x^{2}} d x d t<+\infty
$$


If $u \in \mathcal{W}_{T}$ (see end of Section 3 ), then we find for all $h \in \mathcal{S}\left(\mathbb{R}^{d+1}\right)$,

$$
\begin{aligned}
\int_{0}^{1} \int_{\mathbb{R}^{d}}|v(t, x ; h)| e^{-k x^{2}} d x d t & =\int_{0}^{t} \int_{\mathbb{R}^{d}}\left|\left\langle u(t, x),: e^{\langle\cdot, h\rangle}:\right\rangle\right| e^{-k x^{2}} d x d t \\
& \leq e^{\frac{1}{2}|h|_{2, p}^{2}} \int_{0}^{t} \int_{\mathbb{R}^{d}}\|u(t, x)\|_{2,-p} e^{-k x^{2}} d x d t<+\infty
\end{aligned}
$$

for appropriately chosen $p \in \mathbb{N}, k>0$. Since a solution of $(3.1,3)$ has an $S$-transform which solves the initial value problem for $(4.1)$, it follows that $(3.1,3)$ has a unique solution in $\mathcal{W}_{T}$. We summarize:

Theorem 4.5. Let $T>0$. Under hypotheses (H1), (H2), and (H3) the Cauchy problem $(3.1,3)$ has a unique solution in the class $\mathcal{W}_{T}$, and the solution is given by $(4.9)$.

\section{The Solution of $D_{t} u=L u+\xi \cdot \nabla u$}

In this section we treat the Cauchy problem (3.2), (3.3). The main idea is similar to that of Section 4 , but this time we use the Girsanov formula instead of the Feynman-Kac formula. Recall that $\xi$ is a $d$-dimensional white noise depending on space $x \in \mathbb{R}^{d}$ and time $t \in \mathbb{R}_{+}$. Accordingly, the $S$-transform has to be defined using $d$-tuple $h=\left(h_{1}, \ldots, h_{d}\right)$ of functions $h_{i} \in \mathcal{S}\left(\mathbb{R}^{d}\right), i=1, \ldots, d$, and we set

$$
X_{h}(w):=\sum_{i=1}^{d}\left\langle w_{i}, h_{i}\right\rangle, \quad w_{i} \in \mathcal{S}^{\prime}\left(\mathbb{R}^{d+1}\right), i=1, \ldots, d
$$

in the definition (2.1) of the $S$-transform. (Of course, in (2.2) $|h|_{2}^{2}$ then stands for $\left.\sum_{i=1}^{d}\left|h_{i}\right|_{L^{2}\left(\mathbb{R}^{d+1}\right)}^{2}\right)$ The $S$-transform of equation (3.1) at $h$ reads $(v:=S u)$ :

$$
D_{t} v(t, x)=L v(t, x)+\sum_{i=1}^{d} h_{i}(t, x) D_{i} v(t, x)
$$

where we have suppressed the dependence of $v$ on $h \in \mathcal{S}\left(\mathbb{R}^{d+1}\right)^{d}$. Again it is well known that under conditions ( $\mathrm{H} 1)$, (H2), the initial value problem for $(5.1)$ with $v(0)=f \in$ $C_{b}\left(\mathbb{R}^{d}\right)$ has a solution $v$, which is bounded and continuous on $[0, T] \times \mathbb{R}^{d}$ for every $T>0$.

First we are going to prove that Lemma 4.1 also holds for the solution of the Cauchy problem under consideration. However, for simplicity we shall only treat the case where $L=\frac{1}{2} \Delta$ : the general case follows by obvious modifications of the argument. We use the Girsanov formula (in a suitable form which can be found, e.g., in [4]) for the solution $v$ above and obtain the following respresentation

$$
v(t, x ; h)=\widetilde{\mathbb{E}}(f(x+\widetilde{B}(t)) G(t, x ; h))
$$

where we have set

$$
G(t, x ; h)=\exp \left(\sum_{i=1}^{d} \int_{0}^{t} h_{i}(t-s, x+\widetilde{B}(s)) d \widetilde{B}_{i}(s)-\frac{1}{2} \sum_{i=1}^{d} \int_{0}^{t} h_{i}(t-s, x+\widetilde{B}(s))^{2} d s\right)
$$


As in the previous section $\widetilde{B}$ is an independent $\mathbb{R}^{d}$-valued Brownian motion on a probability space $(\widetilde{\Omega}, \widetilde{\mathcal{B}}, \widetilde{P})$, and $\widetilde{\mathbb{E}}$ denotes the expectation with respect to $\widetilde{P}$. It is quite obvious that replacing $h$ in (5.2) with $g+z h, h, g \in \mathcal{S}\left(\mathbb{R}^{d+1}\right)^{d}, z \in \mathbb{C}$, leads to an entire function $z \mapsto v(t, x ; g+z h)$. We have to estimate $|v(t, x ; z h)|$. Note first that

$$
|G(t, x ; z h)|=G(t, x ; \operatorname{Re} z h) \exp \left(\frac{1}{2}(\operatorname{Im} z)^{2} \sum_{i=1}^{d} \int_{0}^{t} h_{i}(t-s, x+\widetilde{B}(s))^{2} d s\right) .
$$

Since $\widetilde{\mathbb{E}}(G(t, x ; \operatorname{Re} z h))=1$ we obtain the bound

$$
|v(t, x ; z h)| \leq|f|_{\infty} e^{\frac{1}{2} t|z|^{2} \sum_{i=1}^{d}\left|h_{i}\right|_{\infty}^{2}}
$$

This is sufficient to conclude that for every $(t, x) \in \mathbb{H}$ there exists $u(t, x) \in(\mathcal{S})^{*}$ so that $S u(t, x)=v(t, x)$.

It is useful for the following argument to note that the preceeding estimation yields immediately the following bound:

$$
\widetilde{\mathbb{E}}\left(|G(t, x ; z h)|^{2}\right) \leq e^{3 t|z|^{2} \sum_{i=1}^{d}\left|h_{i}\right|_{\infty}^{2}} .
$$

In order to prove that $u$ is weakly continuously differentiable to order 1 in $t$ and to order 2 in $x$, we have to estimate the corresponding derivatives of $v(t, x ; z h)$ locally in a uniform way. Using (5.2), (5.3) and Itô calculus we get the following formula:

$$
\begin{aligned}
D_{t} v(t, x ; z h)=\widetilde{\mathbb{E}} & \left(H_{0}(t, x ; z h) f(x+\widetilde{B}(t)) G(t, x ; z h)\right)+\widetilde{\mathbb{E}}(L f(x+\widetilde{B}(t)) G(t, x ; z h)) \\
& +z \sum_{i=1}^{d} \widetilde{\mathbb{E}}\left(h_{i}(0, x+\widetilde{B}(t)) D_{i} f(x+\widetilde{B}(t)) G(t, x ; z h)\right),
\end{aligned}
$$

with

$$
\begin{aligned}
H_{0}(t, x ; h)=\sum_{i=1}^{d} & {\left[\int_{0}^{t}\left(D_{0} h_{i}\right)(t-s, x+\widetilde{B}(s)) d \widetilde{B}_{i}(s)-\frac{1}{2} h_{i}(0, x+\widetilde{B}(t))^{2}\right.} \\
& -\int_{0}^{t} h_{i}(t-s, x+\widetilde{B}(s)) D_{0} h_{i}(t-s, x+\widetilde{B}(s) d s]
\end{aligned}
$$

With Schwarz' inequality, (5.4), and the Itô isometry it is easy to show that for every $T>0$ there exists a constant $K_{T}>0$ so that for all $t \in[0, T], z \in \mathbb{C}, h \in \mathcal{S}\left(\mathbb{R}^{d+1}\right)^{d}$,

$$
\left|D_{t} v(t, x ; z h)\right| \leq K_{T}\left(|f|_{\infty}+|L f|_{\infty}+\sum_{i=1}^{d}\left|D_{i} f\right|_{\infty}\right) e^{K_{T}\left(1+|z|^{2}\right) \sum_{i=1}^{d}\left(\left|h_{i}\right|_{\infty}^{2}+\left|D_{0} h_{i}\right|_{\infty}^{2}\right)}
$$

Therefore an application of Lemma A.1.3 in [11] proves that $u(t, x)$ is weakly continuously differentiable in $t$ and $S\left(D_{t} u\right)=D_{t} v$. 
For $D_{i} v, i=1, \ldots, d$, we get the following expression

$$
D_{i} v(t, x ; z h)=\widetilde{\mathbb{E}}\left(H_{i}(t, x ; z h) f(x+\widetilde{B}(t)) G(t, x ; z h)\right)+\widetilde{\mathbb{E}}\left(D_{i} f(x+\widetilde{B}(t)) G(t, x ; z h)\right),
$$

with

$$
\begin{aligned}
H_{i}(t, x ; h)=\sum_{j=1}^{d} & \left(\int_{0^{\prime}}^{t} D_{i} h_{j}(t-s, x+\widetilde{B}(s)) d \widetilde{B}_{j}(s)\right. \\
& \left.-\int_{0}^{t} h_{j}(t-s, x+\widetilde{B}(s)) D_{i} h_{j}(t-s, x+\widetilde{B}(s)) d s\right) .
\end{aligned}
$$

The same arguments as above lead to an estimate of the form

$$
\left|D_{i} v(t, x ; z h)\right| \leq K_{t}\left(|f|_{\infty}+\left|D_{i} f\right|_{\infty}\right) e^{K_{t}\left(1+|z|^{2}\right) \sum_{j=1}^{d}\left(\left|h_{j}\right|_{\infty}^{2}+\left|D_{i} h_{j}\right|_{\infty}^{2}\right)}
$$

where the constant $K_{t}$ only depends on $t$; - in particular the estimate is uniform in $x \in$ $\mathbb{R}^{d}$. Therefore we obtain in the same way as before that $u(t, x)$ is weakly continuously differentiable with respect to $x$ and for all $i=1, \ldots, d,(t, x) \in \mathbb{H}, S\left(D_{i} u\right)(t, x)=D_{i} v(t, x)$.

Finally, the second order terms $D_{i} D_{j} v(t, x)$ do not present any new difficulties, and are left as an exercise to the interested reader. Hence we have established the statement of Lemma 4.1 for the current case. The additional arguments which led to Theorem 4.2 and 4.4 can be taken over without any change. Altogether we established the following result:

Theorem 5.1. Under conditions (H1), (H2) the initial value problem (3.2), (3.3) with $f \in C_{b}^{2}\left(\mathbb{R}^{d}\right)$, has a solution $u(t, x)$, which for every $T>0$ is a bounded, weakly continuous mapping from $[0, T] \times \mathbb{R}^{d}$ into $(\mathcal{S})^{*}$. Moreover, this solution is given by the inverse $S$ transform of (5.2). If for $T>0$ in addition (H3) holds, then this solution is unique in the $\operatorname{class} \mathcal{W}_{T}$.

\section{References.}

[1] F.E. Benth, Th. Deck and J. Potthoff, Nonlinear evolution equations with gradient coupled noise, Preprint Nr. 194/95, Mannheim University, 1995

[2] P.L. Chow, Generalized solution of some parabolic equations with random drift, J. Appl. Math. Optimization 20 (1989) 81 96

[3] Th. Deck and J. Potthoff, On a stochastic partial differential equation related to turbulent transport, Preprint Nr. 189/95, Mannheim University, 1995

[4] M. Freidlin, Functional Integration and Partial Differential Equations. Princeton Univ. Press, Princeton (1985)

[5] A. Friedman, Partial Differential Equations of Parabolic Type. Krieger, Malabar (1983)

[6] T. Hida, H.-H. Kuo, J. Potthoff and L. Streit, White Noise - An Infinite Dimensional Calculus. Kluwer Academic Publishers. Dordrecht (1993) 
[7] H. Holden, T. Lindstrøm, B. Øksendal, J. Ubøe and T.-S. Zhang, The Burgers equation with a noisy force and the stochastic heat equation, Commun. PDE 19 (1994) 119-141

[8] Yu.G. Kondratiev, P. Leukert, J. Potthoff, L. Streit and W. Westerkamp, Generalized functionals in Gaussian spaces - The characterization theorem revisited, Preprint Nr. 175/94, Mannheim University, 1994, to appear in J. Funct. Anal.

[9] R. Mikulvicus and B.L. Rozovskii, Soft solution of linear parabolic SPDE's and the Wiener expansion, in: Stochastic analysis on infinite dimentional spasces, H. Kunita and H.-H. Kuo (ed.'s). Longman Scientific and Technical, Harlow (1994)

[10] D. Nualart and M. Zakai, Generalized Brownian functionals and the solution to a stochastic partial differential equation, J. Funct. Anal. 84 (1989) 279-296

[11] J. Potthoff, White noise approach to parabolic stochatic partial differential equations, in: Stochastic Analysis and Applications in Physics, A. I. Caradoso et al. (ed.'s). Kluwer Academic Publishers, Dordrecht (1994) 